09

\title{
Генерация высоковольтных импульсов карбидкремниевыми дрейфовыми диодами с резким восстановлением (сравнение диодов с базой $n$ - и $p$-типа)
}

\author{
(C) П.А. Иванов, И.В. Грехов \\ Физико-технический институт им. А.Ф. Иофффе РАН, \\ 194021 Санкт-Петербург, Россия \\ e-mail: Pavel.Ivanov@mail.ioffe.ru
}

(Поступило в Редакцию 11 мая 2017 г.)

\begin{abstract}
Проведены расчеты временных характеристик импульсных генераторов с дрейфовыми диодами с резким восстановлением на основе $4 \mathrm{H}$-SiC. Показано, что при фиксированных величинах амплитуды и начального „пьедестала“ на зависимости выходного напряжения (не более 5\% от амплитуды) 4H-SiC-диоды с базой $p$-типа уступают диодам с базой $n$-типа по быстродействию.
\end{abstract}

DOI: $10.21883 /$ JTF.2018.01.45488.2327

\section{Введение}

В импульсной электронике широко применются импульсные генераторы с индуктивным накоплением энергии и последующей передачей энергии в нагрузку с помощью быстродействующих размыкателей тока. В таких генераторах (рис. 1) ток $I$, первоначально протекающий через накопительную индуктивность $L$ и замкнутый ключ $S$, при размыкании ключа переходит в параллельную нагрузку $R$, формируя на ней быстронарастающий импульс напряжения $U$.

В настоящее время в качестве быстродействующих размыкателей тока широко используются дрейфовые диоды с резким восстановлением (ДДРВ) [1]. Принцип работы ДДРВ кратко сводится к следующему. Вначале через диод пропускается импульс тока в прямом направлении, во время которого базовые области диода заполняются неравновесными носителями, инжектированными из эмиттеров (фаза накачки). Затем диод переключается в обратное направление и начинается вытягивание неравновесных носителей нарастающим обратным током (фаза выкачки). В тот момент, когда концентрация неравновесных носителей у $p$-n-перехода становится меньше концентрации примесей в блокирущей базе, наступает фаза обрыва обратного тока, сопровождющаяся расширением области пространственного заряда (ОП3) в базу. Обрыв тока происходит максимально быстро, если к началу расширения ОПЗ 1) неравновесные носители из базовых областей выведены полностью (чтобы добиться этого, конструкция диода, а также параметры накачки и выкачки неравновесных носителей подбираются, исходя из подвижностей и времен жизни инжектированных носителей), 2) обратный ток достаточно большой по величине для быстрой перезарядки емкости $p-n$-перехода. Иными словами, для максимального быстродействия ДДРВ необходимо обеспечить такие условия, при которых барьерная емкость блокирующего $p-n$-перехода перезаряжается большим по величине начальным током смещения.
Ранее генерация импульсов карбидкремниевыми ДДРВ была промоделирована нами с учетом влияния на переходной процесс обратного восстановления диода нелинейного сопротивления квазинейтральной части блокирующей базы [2]. Моделирование показало, что на параметры генерируемых импульсов существенным образом влияет вид полевой зависимости скорости дрейфа носителей тока. В $4 H-\mathrm{SiC}$ характеристики дрейфа электронов и дырок сильно отличаются. В настоящей работе проведено моделирование генерации импульсов карбидкремниевыми ДДРВ с базой $n$ - и $p$-типа с целью сравнения их по быстродействию.

\section{Постановка задачи}

В ходе последующего анализа мы будем считать, что к моменту начала расширения ОПЗ (при $t=0$ ) неравновесных носителей в диоде нет, а протекающий через диод ток - это начальный ток смещения, перезаряжающий барьерную емкость $p-n$-перехода. Экви-

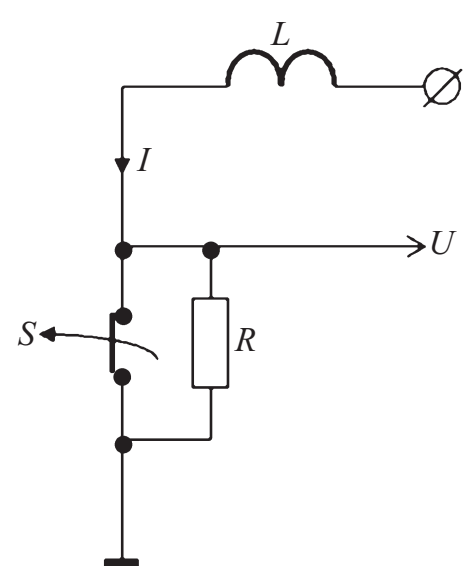

Рис. 1. Базовый принцип работы генератора импульсов с индуктивным накопителем энергии. 


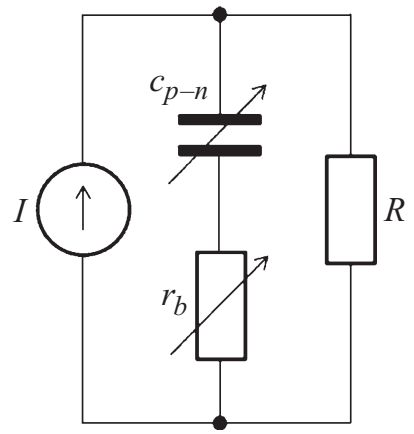

Рис. 2. Эквивалентная схема генератора на стадии обрыва диодом обратного тока.

валентная схема генератора на стадии обрыва диодом обратного тока показана на рис. 2. На этой схеме: $I$ - источник квазипостоянного тока, поддерживаемого накопительной индуктивностью (на схеме не показана); $c_{p-n}(t)$ и $r_{b}(t)$ - мгновенные значения барьерной емкости $p-n$-перехода и последовательного сопротивления квазинейтральной части базы соответственно; $R-$ сопротивление нагрузки (все расчеты выполнялись для $R=50 \Omega$ ). Как было сказано выше, при $t=0$ плотность обрываемого диодом обратного тока $j_{p-n}(0)$ выбирают достаточно большой для того, чтобы минимизировать площадь $p-n$-перехода $S$ и соответственно его барьерную емкость $c_{p-n}$. Однако вместе с ростом $j_{p-n}(0)$ и уменьшением $S$ будет увеличиваться и сопротивление $r_{b}(0)$. В свою очередь большая величина $r_{b}(0)$ будет приводить к возникновению так называемого „пьедестала“ - начального напряжения $u(0)$ - на выходном импульсе

$$
u(0)=I R \frac{r_{b}(0)}{R+r_{b}(0)} .
$$

Если $r_{b}(0) \sim R$, то $u(0)$ может составлять значительную долю от полной амплитуды выходного импульса $(U=I R)$, что является нежелательным (и часто недопустимым) для практических применений генераторов. Задача последующего моделирования состоит в том, чтобы рассчитать временные параметры таких генераторов, которые обеспечивают заданную амплитуду выходных импульсов при величине „пьедестала“ не более 5\% от амплитуды. Согласно выражению (1), при $R=50 \Omega$ и $k=u(0) / U=0.05$ сопротивление $r_{b}(0)$ должно составлять

$$
r_{b}(0)=\frac{k}{1-k} R \approx k R=2.5 \Omega .
$$

\section{Моделирование}

В дальнейших расчетах мы будем использовать следующую распространенную аппроксимацию зависимости дрейфовой скорости носителей $v$ от электри- ческого поля $E$ :

$$
v=\frac{\mu E}{\left[1+\left(\frac{\mu E}{v_{s}}\right)^{\gamma}\right]^{\frac{1}{\gamma}}},
$$

где $\mu$ - низкополевая подвижность носителей, $v_{s}-$ насыщенная скорость дрейфа носителей, $\gamma$ - безразмерный параметр.

В работе [3] нами были проведены исследования дрейфа электронов в $4 H$-SiC $n$-типа (в направлении гексагональной оси $C$ кристалла) при полях вплоть до $4 \cdot 10^{5} \mathrm{~V} / \mathrm{cm}$. Была получена полуэмпирическая зависимость скорости дрейфа электронов $v_{n}$ от поля $E$ (рис. 3). При уровне легирования донорами $\sim 10^{15} \mathrm{~cm}^{-3}$ числовые значения параметров следующие: низкополевая подвижность электронов $\mu_{n}=950 \mathrm{~cm}^{2}(\mathrm{~V} \cdot \mathrm{s})$, насыщенная скорость дрейфа $v_{s n}=1.55 \cdot 10^{7} \mathrm{~cm} / \mathrm{s}$ (полученная величина скорости насыщения оказалась близкой к значению, полученному в результате численного моделирования методом Монте-Карло [4]), безразмерный параметр $\gamma_{n}=1.15$.

Для 4H-SiC $p$-типа надежных экспериментальных данных относительно указанных выше параметров пока нет (транспорт горячих дырок в $4 H-\mathrm{SiC}$ исследовался до настоящего времени исключительно моделированием - методом Монте-Карло [5]). Аппроксимация теоретической зависимости $v_{p}$ от $E$, приведенная в [5] (рис. 3), дала следующие значения числовых параметров: $\mu_{p}=70 \mathrm{~cm}^{2}(\mathrm{~V} \cdot \mathrm{s}), v_{s p}=5.9 \cdot 10^{6} \mathrm{~cm} / \mathrm{s}, \gamma_{p}=1.18$.

С учетом формулы (3) сопротивление $r_{b}(0)$ вычисляется следующим образом:

$$
r_{b}(0)=r_{b o}\left[1+\left(\frac{\mu k U}{L_{b} v_{s}}\right)^{\gamma}\right]^{\frac{1}{\gamma}},
$$

где $r_{b o}=\rho L_{b} / S$ - омическое сопротивление базы $(\rho=q n \mu-$ удельное сопротивление базы, $q-$ элемен-

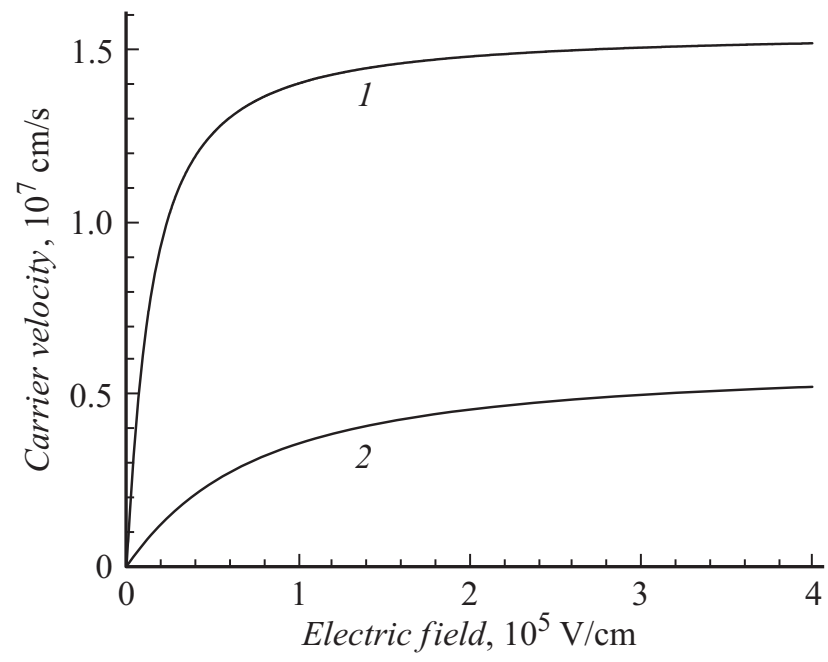

Рис. 3. Полевые зависимости дрейфовой скорости носителей в $4 H$-SiC: 1 - электроны [3], 2 - дырки [5]. 
тарный заряд, $n-$ концентрация свободных носителей в базе, $L_{b}$ - толщина базы).

Выберем следующие условия для расчетов:

1) амплитуда выходного импульса на нагрузке близка к напряжению пробоя диода (в высоковольных 4H-SiCдиодах эффективность охранной системы обычно такова, что максимальное обратное напряжение не превышает 90\% от теоретического напряжения пробоя квазиодномерного перехода $\left(U_{b r}\right)$ :

$$
U=0.9 \cdot U_{b r}
$$

2) толщина базы $\left(L_{b}\right)$ равна толщине ОПЗ при обратном напряжении, равном $U_{b r}$ :

$$
L_{b}=\sqrt{\frac{2 \varepsilon U_{b r}}{q N}}
$$

где $\varepsilon=8.85 \cdot 10^{-13} \mathrm{~F} / \mathrm{cm}$ - диэлектрическая проницаемость $4 H-\mathrm{SiC}, N-$ концентрация примесей в блокирующей базе.

При данных условиях напряжение $U_{b r}$ и концентрация $N$ связаны соотношением

$$
U_{b r}=\frac{\varepsilon E_{b r}^{2}}{2 q N}
$$

где $E_{b r}-$ критическая напряженность поля лавинного пробоя $4 H-\mathrm{SiC}$. Величина $E_{b r}$ связана с концентрацией примесей $N$ в блокирующей базе следующим эмпирическим соотношением [6]:

$$
E_{b r}\left(\frac{\mathrm{V}}{\mathrm{cm}}\right)=\frac{2.49 \cdot 10^{6}}{1-0.25 \cdot \log \left(\frac{N\left(\mathrm{~cm}^{-3}\right)}{10^{16}}\right)} .
$$

Выражения (7) и (8) позволяют для наперед заданного напряжения $U$ рассчитать (численным методом) концентрацию $N$. В свою очередь толщина базы $L_{b}$ диода рассчитывается по формуле (6).

Отличительной особенностью $4 H$-SiC $p$-типа является неполная ионизация акцепторов (алюминий) при комнатной температуре вследствие относительно большой энергии их ионизации: $E_{A}=0.19 \mathrm{eV}$. (Доноры (азот) в $4 H-\mathrm{SiC}$, имеющие энергию ионизации около $0.05 \mathrm{eV}$, при комнатной температуре ионизированы практически полностью).

Очевидно, что вследствие неполной ионизации акцепторов и сравнительно малой подвижности дырок площадь диодов с $p$-базой должна быть больше по сравнению с диодами с n-базой (при фиксированной амплитуде выходных импульсов).

В дальнейших расчетах концентрация электронов в диодах с $n$-базой принималась равной концентрации доноров $\left(n_{n}=N_{D}\right)$, а концентрация дырок в диодах с $p$-базой рассчитывалась из стандартного уравнения нейтральности:

$$
n_{p}=N_{v} \exp \left(-\frac{E_{F}}{k T}\right) \frac{N_{A}}{1+2 \exp \left(\frac{E_{A}-E_{F}}{k T}\right)},
$$

где $N_{v}$ - эффективная плотность состояний в валентной зоне $4 H-\mathrm{SiC}, k T-$ тепловая энергия, $E_{F}-$ положение уровня Ферми в запрещенной зоне $\mathrm{p}-4 \mathrm{H}-\mathrm{SiC}$ относительно потолка валентной зоны.

Введенные и рассчитанные выше параметры позволяют определить требуемую площадь перехода $S$ :

$$
S=\frac{L_{b}}{q n \mu} \frac{1}{k R}\left[1+\left(\frac{\mu k U}{L_{b} v_{s}}\right)^{\gamma}\right]^{\frac{1}{\gamma}} .
$$

В соответствии со схемой на рис. 2 мгновенная величина напряжения описывается дифференциальным уравнением

$$
u_{p-n}+c_{p-n} \frac{d p_{p-n}}{d t}\left(R+r_{b}\right)=U .
$$

В уравнении (10) нелинейная емкость $c_{p-n}(t)$ уменьшается со временем вследствие расширения ОПЗ в базу. Нелинейное сопротивление $r_{b}(t)$ максимально в начале фазы обрыва тока и уменышается со временем как за счет расширения ОПЗ, так и за счет ослабления эффекта насыщения скорости носителей. Начальное условие для решения уравнения $(10): u_{p-n}(0)=0$. В уравнении $(10)$ члены $c_{p-n}$ и $r_{b}$ могут быть выражены через напряжение $u_{p-n}$ и производную $d u_{p-n} / d t$ следующим образом.

Зависимость емкости $c_{p-n}$ от напряжения $u_{p-n}$ :

$$
c_{p-n}=S \sqrt{\frac{q \varepsilon N}{2\left(u_{p-n}+U_{b i}\right)}},
$$

где $U_{b i}-$ диффузионная разность потенциалов $p-n$-перехода.

Сопротивление квазинейтральной части базы:

$$
r_{b}=\frac{\rho w_{b}}{S}\left\{1+\left[\frac{\mu u_{r}}{w_{b} v_{s}}\right]^{\gamma}\right\}^{\frac{1}{\gamma}} .
$$

Падение напряжения на квазинейтральной части базы и ее толщина

$$
u_{r}=U_{m}-u_{p-n}-R c_{p-n} \frac{d u_{p-n}}{d t} ; \quad w_{b}=L_{b}-\frac{\varepsilon S}{c_{p-n}} .
$$

Искомая форма выходного импульса, т. е. зависимость $u(t)=u_{p-n}(t)+u_{r}(t)$, определялась $\mathrm{c}$ помощью комбинации численных методов, включающей численное решение дифференциальных уравнений первого порядка и численное решение трансцендентных алгебраических уравнений.

На рис. 4 показаны временные зависимости выходного напряжения, рассчитанные при разных значениях амплитуды $U$. Как видно, при амплитуде $U=1000 \mathrm{~V}$ длительность фронта нарастания импульса в случае диода с $n$-базой составляет (по уровню $0.1-0.9$ ) около $0.1 \mathrm{~ns}$, в то время как в случае $p$-базового диода длительность фронта больше почти в десять раз. Диоды с $n$-базой 


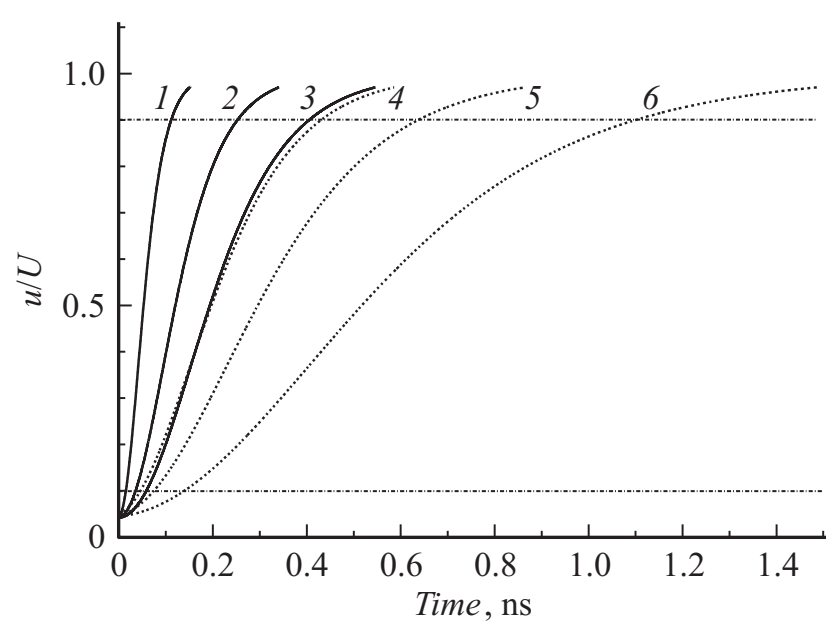

Рис. 4. Временные зависимости выходного напряжения, рассчитанные при разных значениях $U$. Сплошные линии - база $n$-типа, пунктирные линии - база $p$-типа. $U(\mathrm{~V}): 1,6-1000$, $2-2000,3-3000,4-300,5-500$.

могут генерировать $3000 \mathrm{~V}$ импульсы с длительностью фронта около $0.3 \mathrm{~ns}$. При том же быстродействии диоды c $p$-базой могут генерировать импульсы амплитудой лишь $300 \mathrm{~V}$.

\section{Заключение}

Проведенные исследования показали, что при фиксированных величинах амплитуды выходных импульсов и начального „пьедестала“ $4 H-\mathrm{SiC}$ ДДРВ с блокирующей базой $p$-типа должны намного уступать ДДРВ с базой $n$-типа по быстродействию. Причиной этого являются меньшие по величине подвижность дырок, концентрация дырок (при одинаковом уровне легирования) и насыщенная скорость их дрейфа.

Исследование выполнено за счет гранта Российского научного фонда (проект№ 14-29-00094).

\section{Список литературы}

[1] Грехов И.В., Месяи, Г.А. // УФН. 2005. Т. 175. № 7. C. $735-744$.

[2] Иванов П.А., Грехов И.В. // ЖТФ. 2016. Т. 86. Вып. 2. C. $85-88$.

[3] Ivanov P.A., Potapov A.S., Samsonova T.P, Grekhov I.V. // Sol. Stat. Electron. 2016. Vol. 123. P. 15-18.

[4] Hjelm M., Nilsson H.-E., Martinez A., Brennan K.F., Bellotti E. // J. Appl. Phys. 2003. Vol. 93. P. 1099-1107.

[5] Bellotti E., Farahmand M., Goano M., Ghillino E., Garetto C., Ghione G., Nilsson H.-E., Brennan K.F., Ruden P.P. // Intern. J. High Speed Electron. and Systems. 2001. Vol. 11. P. 525-584.

[6] Konstantinov A.O., Wahab Q., Nordell N., Lindefelt U. // Appl. Phys. Lett. 1997. Vol. 71. N 1. P. 90-92. 